\title{
Identifying Quantitative Trait Loci for Resistance to Sclerotinia Head Rot in Two USDA Sunflower Germplasms
}

\author{
B. Yue, S. A. Radi, B. A. Vick, X. Cai, S. Tang, S. J. Knapp, T. J. Gulya, J. F. Miller, and J. Hu
}

First and fourth authors: Department of Plant Sciences, North Dakota State University, Fargo 58105; second author: Dow AgroSciences, 2230 Highway 75 North, Breckenridge, MN 56560; third, seventh, eighth, and ninth authors: U.S. Department of Agriculture, Agricultural Research Service, Northern Crop Science Laboratory, Fargo, ND 58105; and fifth and sixth authors: Center for Applied Genetic Technologies, The University of Georgia, Athens 30602.

Accepted for publication 3 April 2008.

\begin{abstract}
Yue, B., Radi, S. A., Vick, B. A., Cai, X., Tang, S., Knapp, S. J., Gulya, T. J., Miller, J. F., and Hu, J. 2008. Identifying quantitative trait loci for resistance to Sclerotinia head rot in two USDA sunflower germplasms. Phytopathology 98:926-931.

Sclerotinia head rot is a major disease of sunflower in the world, and quantitative trait loci (QTL) mapping could facilitate understanding of the genetic basis of head rot resistance and breeding in sunflower. One hundred twenty-three $F_{2: 3}$ and $F_{2: 4}$ families from a cross between HA 441 and RHA 439 were studied. The mapping population was evaluated for disease resistance in three field experiments in a randomized complete

ity (DS) were assessed. A genetic map with 180 target region amplification polymorphism, 32 simple sequence repeats, 11 insertion-deletion, and 2 morphological markers was constructed. Nine DI and seven DS QTL were identified with each QTL explaining 8.4 to $34.5 \%$ of phenotypic variance, suggesting the polygenic basis of the resistance to head rot. Five of these QTL were identified in more than one experiment, and each QTL explained more than $12.9 \%$ of phenotypic variance. These QTL could be useful in sunflower breeding. Although a positive correlation existed between the two disease indices, most of the respective QTL were located in different chromosomal regions, suggesting a different genetic basis for the two indices.
\end{abstract} block design with two replicates. Disease incidence (DI) and disease sever-
Sunflower (Helianthus annuus L.) is one of the staple oilseed crops of the world. The ubiquitous Sclerotinia sclerotiorum (Lib.) de Bary attacks all parts of the sunflower plant and causes three distinct diseases: head rot, basal stalk rot, and mid-stalk rot. It is one of the most important pathogens worldwide. In the northern Great Plains of the United States, head rot has been the greatest threat to sunflower production. The incidence of head rot can increase drastically under a high rainfall condition during the flowering stage. In 1999, more than $80 \%$ of the sunflower fields in eastern North Dakota had high incidences of head rot causing an estimated 70 million dollar loss (5). Breeding for resistant hybrids is an important approach to reduce the damage from $S$. sclerotiorum since chemical control is difficult and not feasible economically (14).

Progress in breeding for head rot resistance is limited due to the lack of effective sources of resistance. Breeding relies on identification and incorporation of partial resistance from diverse genotypes. Evaluation of head rot resistance in the field is difficult since environmental factors play a significant role in disease development. However, molecular markers tightly linked to head rot resistance genes can be employed to assist the selection for resistance genes during variety development. The advent and development of molecular markers and genetic maps have facilitated understanding of the genetic basis of head rot resistance. About a dozen linkage maps have been published using different molecular markers, including restriction fragment length polymorphism (RFLP), random amplification of polymorphic DNA

Corresponding author: J. Hu; E-mail address: jinguo.hu@ars.usda.gov

doi:10.1094/PHYTO-98-8-0926

This article is in the public domain and not copyrightable. It may be freely reprinted with customary crediting of the source. The American Phytopathological Society, 2008.
(RAPD), amplified fragment length polymorphism (AFLP), and simple sequence repeats (SSR) in sunflower $(4,20)$. In addition, Lai et al. (9) added 243 markers derived from expressed sequence tags (EST) to the sunflower SSR map. Mestries et al. (14) identified three quantitative trait loci (QTL) for head rot resistance, each explaining 12.3 to $17.5 \%$ of phenotypic variance. Measured by percentage attack and the number of days between infection and the appearance of symptoms (latency index) in two populations, Bert et al. $(1,2)$ identified 20 QTL for head rot resistance. Rönicke et al. (18) detected five QTL governing head rot resistance, and each explained 10.6 to $17.1 \%$ of the total phenotypic variance.

Recently, a polymerase chain reaction (PCR)-based target region amplification polymorphism (TRAP) marker technique was developed to take advantage of the annotated EST as a reference to generate polymorphic markers (6). This marker technique reveals high levels of polymorphisms in various crops $(15,22)$, and over 400 TRAP markers have been added to the public sunflower linkage map (8). This study reports on the construction of a linkage map primarily using TRAP in an $\mathrm{F}_{2}$ sunflower population and the result of QTL mapping for resistance to head rot in two U.S. Department of Agriculture (USDA)-released sunflower germplasms.

\section{MATERIALS AND METHODS}

Plant materials and disease evaluation. Two oilseed-type sunflower breeding lines, HA 441 (PI 639164) and RHA 439 (PI 639162), with resistance to head rot, were recently released by the USDA-ARS and the North Dakota Agricultural Experimental Station (16). HA 441 is a cytoplasmic male sterility maintainer line and RHA 439 is a restorer line. One hundred and twentythree $F_{2: 3}$ and $F_{2: 4}$ families from a cross between HA 441 and RHA 439 , as well as the two parents, were used in field inoculation 
tests in the summer of 2006 and 2007. Seeds of the $F_{2: 3}$ families were harvested from 123 selfed $\mathrm{F}_{2}$ individuals, and the seed of the $\mathrm{F}_{2: 4}$ families were the bulk of the seed from six randomly selfed individuals in each $\mathrm{F}_{2: 3}$ family. The $\mathrm{F}_{2: 3}$ and $\mathrm{F}_{2: 4}$ families (20 to 25 plants each) were planted at two field locations (Carrington and Fargo, ND) in a randomized complete block design with two replicates. At the flowering stage, approximately 12 heads in each plot were inoculated with a suspension of 5,000 ascospores per milliliter of water and $5 \mathrm{ml}$ per head. Ascospores were applied directly to the face of individual heads with a hand spray bottle. A lab-grown strain of sclerotia (WM 274), which originally came as ascospores from Nebraska in 1999, was used throughout the study due to its consistent ability to form apothecia. Ascospores were produced with our in-house procedure: sclerotia were produced on cornmeal/sand (20:1, vol/vol). Uniform sized sclerotia were selected for conditioning with three repeated freeze $(24 \mathrm{~h}$ at $\left.-20^{\circ} \mathrm{C}\right) /$ thaw $\left(24 \mathrm{~h}\right.$ at $\left.22^{\circ} \mathrm{C}\right)$ cycles. The conditioned sclerotia were surface sterilized and were placed in petri dishes filled with moistened, sterile silica sand. The dishes were placed under fluorescent illumination (half "cool white" bulbs and half "near UV" bulbs) with a 12 -h photoperiod, held at 15 to $17^{\circ} \mathrm{C}$. Once stipes began to appear, those germinated sclerotia were transferred to water agar plates until the apothecia matured. Ascospore release was triggered by momentarily lifting the lid. The lids with adhering ascospores were removed daily, and stored at $-80^{\circ} \mathrm{C}$ until needed.

To create an effective microenvironment for disease development, a misting system was installed and misted for 5 min after inoculation for $5 \mathrm{~min}$ every half-hour for 3 weeks. Five weeks after the inoculation, disease was scored on individual heads using a scale of 0 through $5(0=$ no symptom; $1=1$ to $20 \%$ rot; $2=21$ to $40 \%$ rot; $3=41$ to $60 \%$ rot; $4=61$ to $80 \%$ rot; and $5=$ 81 to $100 \%$ rot). Disease resistance was evaluated by disease incidence (DI), calculated as the percentage of plants infected within a row, and disease severity (DS), measured by the average disease score of the infected plants within each plot. The test in Fargo was severely damaged by a storm in 2006 . Thus, the data from three tests, Carrington in 2006 (test 1), Carrington in 2007 (test 2), and Fargo in 2007 (test 3) were used for QTL analysis in this study.

DNA preparation. Total genomic DNA was isolated from $50 \mathrm{mg}$ (fresh weight) young leaf tissue sampled from 15 plants of each $\mathrm{F}_{2: 3}$ family using the Qiagen DNeasy 96 Plant Kit (QIAGEN,
Valencia, CA), following the manufacturer's instruction. DNA concentrations were determined with a DU7400 spectrophotometer (Beckman Coulter) and adjusted to $10 \mathrm{ng} / \mu \mathrm{l}$ for PCR amplification.

Genotyping. The TRAP assays followed the procedures described by $\mathrm{Hu}$ and Vick (6) except for the change of the annealing temperature in the first five cycles from 35 to $40^{\circ} \mathrm{C}$ and in the last 30 cycles from 50 to $53^{\circ} \mathrm{C}$. The fixed primers were designed against the expressed sequence tag (EST) sequences in the Compositae Genomics Initiative database, and selected cloned genes in the database of the National Center for Biotechnology Information. A total of 29 fixed primers designed against the EST primarily related to the conserved regions of leucine rich repeat (LRR), nucleotide binding site (NBS), kinase, and telomere were used to generate TRAP markers for map construction. We designed nine arbitrary primers labeled with either IR (infrared) 700 or IR 800 dye for TRAP analysis (6). Sequences of these primers are given in Table 1. The nomenclature of TRAP markers followed the rule of the code of fixed primer + the code of labeled primer + fragment size. In addition, two morphological markers, anthocyanin pigmentation in hypocotyls $(H c)$ and branching $(B)$, were visually evaluated at seedling emergence and flowering stages, respectively.

To assign the linkage groups to previously published SSR maps, 220 SSR and insertion-deletion (INDEL) mapped markers distributed throughout the genome (23) were screened for polymorphisms between the two parents. Of these, 45 were polymorphic and were genotyped in the $F_{2}$ population. SSR and INDEL marker assays were conducted following the procedures described by Tang et al. (20).

Data analysis, map construction and QTL analysis. Analysis of variance (ANOVA) and correlation analysis were performed by the procedures of generalized linear modeling (GLM) and correlation (CORR) using the SAS program (19). The program Mapmaker/EXP 3.0 (11) was used to construct the genetic map (with the Kosambi map function). The means of the data from the two replications were used to detect QTL using the software of Mapmaker/QTL $1.1(10,12)$.

\section{RESULTS AND DISCUSSION}

Phenotypic variance among parents and the $F_{2: 3}$ and $F_{2: 4}$ populations. The phenotypic differences between the parents as

TABLE 1. Source expressed sequence tags identity (EST ID) and accession number and sequence information of some of the fixed and arbitrary primers used for genotyping

\begin{tabular}{lll}
\hline Code & EST ID or GenBank accession no. & Sequences 5'-3' $^{\prime}$ \\
\hline Fixed primers & & \\
T02 & QHA10B18 & GTTTGCCTTTAAGAACCG \\
T05 & QHA11D14 & ATACCCACCCGTCACTAC \\
T08 & QHA11124 & CGGTATAGCCTGTTTGTG \\
T10 & QHA12P24 & CTCCAGTCTGACCCGTTG \\
T12 & QHA13J07 & GGCAAGTCATATGCACGA \\
T17 & QHA20I01 & CCGAGTTGGTATGCTTGT \\
T29 & QHB14G14 & CTCGATAACATCCTCCCA \\
T30 & QHB14G14 & AATCTCAAGGACAAAAGG \\
T36 & QHB18I19 & CTGCCAAGTGAAACGCT \\
T47 & QHF15O11 & CTGGAGCCAAGACATCTG \\
T61 & HAU91339 & ACCATTCAATATCTCATTG \\
T74 & QHL12D20 & CGAGAAAGCACATTCGTTA \\
T75 & QHL12D20 & CCCGTATGAATCCAAGAGTA \\
T83 & QHK11F05 & GAACCAAACTGGGGTATGTA \\
T123 & AT2G44990 & ACGTTATGAGCCCCATGAAGA \\
Arbitrary primers & & \\
R03 & TRAP03(IR-700) & CGTAGCGCGTCAATTATG \\
R19 & SA12(IR-700) \\
R20 & SA14(IR-700) & TTCTAGGTAATCCAACAACA \\
R21 & SA4(IR-700) & TTACCTTGGTCATACAACATT \\
R13 & TRAP13(IR-800) \\
R23 & GA5(IR-800) & TTCTTCTTCCCTGGACACAAA \\
\hline
\end{tabular}


well as the variance in the populations are summarized in Table 2. HA 441 had higher DI and DS than RHA 439 in test 1. Results, however, were reversed in test 2 and test 3 , with a significantly higher DI in RHA 439 than in HA 441. These results suggested the two parents responded differently to the pathogen under different environments. The mean DI was highest in test 3 , and lowest in test 2, while the mean DS did not fluctuate much in all three tests. Transgressive segregation was observed for DI and DS in the populations. The values of skewness and kurtosis for the traits were less than or close to 1.0 except for DS in test 1 . This indicated the traits generally fit to a normal distribution (Table 2).

ANOVA of the data from different tests revealed that variance due to genotypic differences was highly significant for both traits. Variances from tests, block, and genotype by test for DI, and variance from genotype by test for DS were also significant (data not shown). This indicated that environments had significant influence on the traits, and similar results were also observed in previous studies (18). DI was more sensitive to environments than DS in this study. This may be explained by considering that disease incidence might be related to disease avoidance/or escape, which is more complex than disease development.

Correlations between DI and DS. Positive and significant correlations were detected between DI and DS in different tests. The coefficients were highest between DI and DS in the same tests ( 0.57 to 0.64$)$, intermediate for DI across tests (0.40 to 0.49 ), and lowest between DI and DS in the different tests $(0.19$ to 0.32).

Polymorphism and map construction. For SSR markers, the polymorphism was low between the two parents, only 45 of the $220(20.5 \%)$ primer pairs screened were polymorphic. Fifty-eight primer combinations in 29 TRAP reactions amplified 263 polymorphic markers. On average each primer combination generated 4.5 polymorphic bands with a range of 1 to 13 . Of the 263 polymorphic markers, 19 were not linked to other markers and 64 markers were not used to construct the genetic map due to their clustering in certain regions on the chromosomes. The current genetic map for detecting head rot resistance QTL contains 180 TRAP, 32 SSR, 11 INDEL, and 2 morphological markers. This map has 19 linkage groups which span $1797.6 \mathrm{cM}$ with an average distance of $8.0 \mathrm{cM}$ between adjacent markers (Fig. 1). The SSR and INDEL markers were distributed on 16 linkage groups, and these markers were assigned to 16 of the 17 linkage groups of the public sunflower SSR map (23), the exception being linkage group 14. The morphological marker $B$ was mapped to the same chromosomal region on linkage group 10 as described by Tang et al. (21). The other morphological marker, $H c$, mapped to linkage group 6, and this locus was different from another locus $(T)$ controlling anthocyanin pigment located on linkage group 11 (17).

QTL mapping. Nine QTL were detected for DI in the three tests and each explained 9.6 to $26.4 \%$ of phenotypic variance (Table 3). However, only QDi4 was found in all three tests and two others were present in two tests. QDi4 explained 14.8 to
$26.4 \%$ of phenotypic variance in the three tests. HA 441 was the source of six of nine DI resistance QTL.

Seven DS resistance QTL were identified in three tests, each explaining 8.4 to $34.5 \%$ of the phenotypic variance. Two QTL, $Q D s 2$ and $Q D s 5$, were detected in two of the three tests. HA 441 and RHA 439 contributed four and three resistance QTL, respectively, for decreasing DS.

Previous studies indicated that head rot resistance was conferred by multiple genes in sunflower $(1,2,14,18)$. The results in this study also support the polygenic nature of resistance. Both parents in this study contributed head rot resistance QTL to their progenies. These results were expected since the two parents showed partial resistance to head rot (16), and previous studies also reported similar findings (1). It is possible, therefore, to develop germplasm lines with higher levels of resistance by pyramiding these resistance QTL from different genotypes.

Although a positive correlation existed between DI and DS in this study, only 6 of the 16 resistance QTL were located in the same chromosomal regions, suggesting a different genetic basis for the two disease indices. Thus, breeding lines with higher levels of resistance to head rot could be developed by pyramiding these QTL governing different disease indices. Moreover, the mean values of DI and DS decreased from 75.1 to $35.3 \%$, and 4.4 to 3.7 with the increasing number of QTL for DI or DS, respectively. Moreover, the mean values of DI and DS in the families that possessed all 5 QTL were 33.9\% and 3.6, respectively (Table 4). Thus, pyramiding resistance QTL for DI and DS could increase the resistance level of breeding lines and cultivars.

QTL congruence and comparison with previous reports. Resistance QTL for DI and DS were found in 13 chromosomal regions of 10 linkage groups (Fig. 1). Three intervals (T123R20_380 to T47R03_180 in the linkage group 2, T29R03_850 to T08R03_810 in the linkage group 9, and T61R23_360 to T36R03_670 in the linkage group 12) harbored resistance QTL for DI and DS. Moreover, DI and DS resistance QTL were consistently found in the T61R23_360-T36R03_670 interval.

It is difficult to compare the resistance QTL detected in this study with previous results due to different molecular markers being employed. However, three resistance QTL detected in this study can be associated with previous QTL through nearby SSR or morphological markers. Rönicke et al. (18) identified a head lesion length (similar to DS) resistance QTL close to the SSR marker ORS613 in linkage group 10. This study defined a resistance QTL for DS in the same chromosomal region. Near the locus harboring the branching gene $\left(b_{1}\right)$, Bert et al. (1) identified two QTL (percentage attack on capitulum and latency index, similar to DI and DS, respectively) and Mestries et al. (14) detected one (capitulum index, similar to DS) QTL for head rot resistance. In this study, a resistance QTL for DI was detected in that chromosomal region in two tests.

The advantages of TRAP markers. In this study, a linkage map was constructed, mainly employing TRAP markers, for culti-

TABLE 2. Measurement of disease incidence and severity values and population parameters for $123 \mathrm{~F}_{2: 3}$ and $\mathrm{F}_{2: 4}$ families from a cross between sunflower (Helianthus annuиs L.) lines HA 441 and RHA 439 in three field experiments

\begin{tabular}{|c|c|c|c|c|c|c|c|}
\hline \multirow[b]{2}{*}{ Traits $^{\mathrm{a}}$} & \multirow[b]{2}{*}{ HA 441} & \multirow[b]{2}{*}{ RHA 439} & \multicolumn{5}{|c|}{ Population } \\
\hline & & & Range & Mean & SD & Skewness & Kurtosis \\
\hline \multicolumn{8}{|c|}{ Disease incidence $(\%)$} \\
\hline Test 1 & 66.7 & 26.7 & $0.0-94.4$ & 43.3 & 23.5 & 0.3 & -0.6 \\
\hline Test 2 & 6.5 & $68.8^{\mathrm{b}}$ & $0.0-90.6$ & 31.3 & 17.6 & 0.5 & 0.1 \\
\hline Test 3 & 33.3 & $85.0^{\mathrm{b}}$ & $0.0-100.0$ & 56.7 & 24.1 & 0.0 & -0.7 \\
\hline \multicolumn{8}{|c|}{ Disease severity } \\
\hline Test 1 & 4.2 & 4.2 & $0.0-5.0$ & 2.6 & 1.1 & -1.5 & 2.4 \\
\hline Test 2 & 2.5 & 4.5 & $0.0-5.0$ & 4.0 & 1.0 & -1.2 & 1.5 \\
\hline Test 3 & 3.4 & 4.9 & $0.0-5.0$ & 3.9 & 1.1 & -1.1 & 0.7 \\
\hline
\end{tabular}

a Test 1 = study at Carrington, ND, in 2006; Test 2 = study at Carrington, ND, in 2007; Test $3=$ study at Fargo, ND, in 2007.

b Difference is significant at the $P=0.01$ level between the two parents. 


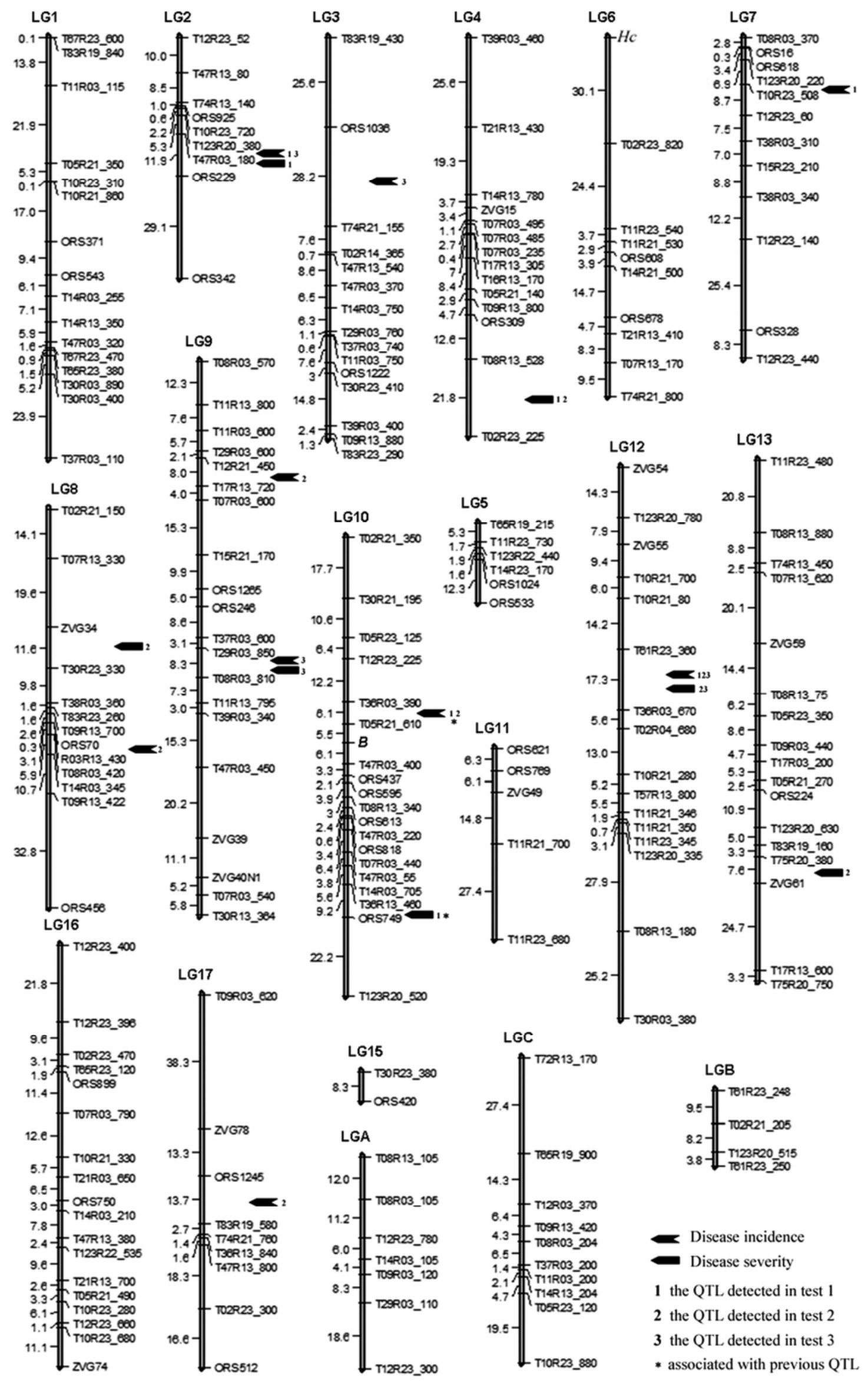

Fig. 1. The location of quantitative trait loci (QTL) for head rot resistance detected in the three tests (test 1, Carrington, ND, in 2006; test 2, Carrington, ND, in 2007; and test 3, Fargo, ND, in 2007). The map is constructed from the HA 441/RHA $439 \mathrm{~F}_{2: 3}$ population. Sixteen simple sequence repeats (SSR)-anchored linkage groups are numbered the same as in the public sunflower map and three groups with only target region amplification polymorphism markers are named A to C. 


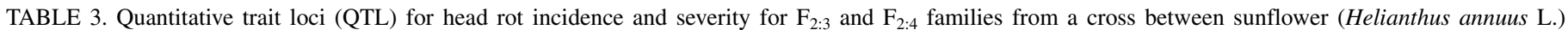
breeding lines HA 441 and RHA 439 using interval mapping

\begin{tabular}{|c|c|c|c|c|c|c|}
\hline Traits $^{\mathrm{a}}$ & QTL & Linkage groups & Marker interval & LOD & $R^{2} \%$ & Resistant parent $^{\mathrm{c}}$ \\
\hline \multicolumn{7}{|c|}{ Disease incidence } \\
\hline \multirow[t]{4}{*}{ Test 1} & QDi1 & 02 & T123R20-380 - T47R03-180 & 3.4 & 13.9 & $\mathrm{H}$ \\
\hline & QDi2 & 07 & T123R20-220 - T10R23-508 & 3.0 & 11.2 & $\mathrm{H}$ \\
\hline & $\widetilde{Q D i 3}$ & 10 & T36R03-390 - T05R21-610 & 3.7 & 14.3 & $\mathrm{R}$ \\
\hline & QDi4 & 12 & T61R23-360 - T36R03-670 & 5.1 & 26.4 & $\mathrm{R}$ \\
\hline \multirow[t]{5}{*}{ Test 2} & $\widetilde{Q D i 5}$ & 08 & ORS70 - R03R13-430 & 5.2 & 17.9 & $\mathrm{R}$ \\
\hline & QDi6 & 09 & T12R21-450 - T17R13-720 & 2.7 & 9.6 & $\mathrm{H}$ \\
\hline & $\widetilde{Q D i 3}$ & 10 & T36R03-390 - T05R21-610 & 3.0 & 12.9 & $\mathrm{R}$ \\
\hline & QDi4 & 12 & T61R23-360 - T36R03-670 & 4.4 & 23.5 & $\mathrm{R}$ \\
\hline & $\widetilde{Q D i 7}$ & 17 & ORS1245 - T83R19-580 & 4.2 & 17.1 & $\mathrm{H}$ \\
\hline \multirow[t]{4}{*}{ Test 3} & QDi1 & 02 & T123R20-380 - T47R03-180 & 5.3 & 18.5 & $\mathrm{H}$ \\
\hline & QDi8 & 03 & ORS1036 - T74R21-155 & 2.8 & 11.1 & $\mathrm{H}$ \\
\hline & QDi9 & 09 & T29R03-850 - T08R03-340 & 3.5 & 16.4 & $\mathrm{H}$ \\
\hline & QDi4 & 12 & T61R23-360 - T36R03-670 & 3.7 & 14.8 & $\mathrm{R}$ \\
\hline \multicolumn{7}{|c|}{ Disease severity } \\
\hline \multirow[t]{3}{*}{ Test 1} & $Q D s 1$ & 02 & T123R20-380 - T47R03-180 & 2.9 & 12.8 & $\mathrm{H}$ \\
\hline & $Q D s 2$ & 04 & T08R13-528 - T02R23-225 & 9.6 & 24.9 & $\mathrm{H}$ \\
\hline & QDs3 & 10 & T36R13-460 - ORS749 & 11.8 & 34.5 & $\mathrm{R}$ \\
\hline \multirow[t]{4}{*}{ Test 2} & $\widetilde{Q D s} 2$ & 04 & T08R13-528 - T02R23-225 & 4.1 & 24.4 & $\mathrm{H}$ \\
\hline & QDs4 & 08 & ZVG34 - T30R23-330 & 2.4 & 8.4 & $\mathrm{R}$ \\
\hline & $Q D s 5$ & 12 & T61R23-360 - T36R03-670 & 4.1 & 22.0 & $\mathrm{R}$ \\
\hline & QDs6 & 13 & T75R20-380 - ZVG61 & 3.5 & 23.9 & $\mathrm{H}$ \\
\hline \multirow[t]{2}{*}{ Test 3} & QDs7 & 09 & T29R03-850 - T08R03-340 & 4.2 & 19.1 & $\mathrm{H}$ \\
\hline & $Q D s 5$ & 12 & T61R23-360 - T36R03-670 & 3.3 & 23.6 & $\mathrm{R}$ \\
\hline
\end{tabular}

a Test 1 = study at Carrington, ND, in 2006; Test 2 = study at Carrington, ND, in 2007; Test 3 = study at Fargo, ND, in 2007.

${ }^{\mathrm{b}}$ Amount of phenotypic variance (\%) explained by the QTL.

${ }^{c}$ The QTL from HA 441(H) or RHA $439(\mathrm{R})$ of the locus had a positive effect on this trait.

TABLE 4. Number of $\mathrm{F}_{2: 3} / \mathrm{F}_{2: 4}$ families from a cross between sunflower (Helianthus annuus $\mathrm{L}$.) breeding lines HA 441 and RHA 439 , and their range and mean disease incidence and disease severity values evaluated in the three field tests that had different number of resistance quantitative trait loci (QTL) detected in more than one test

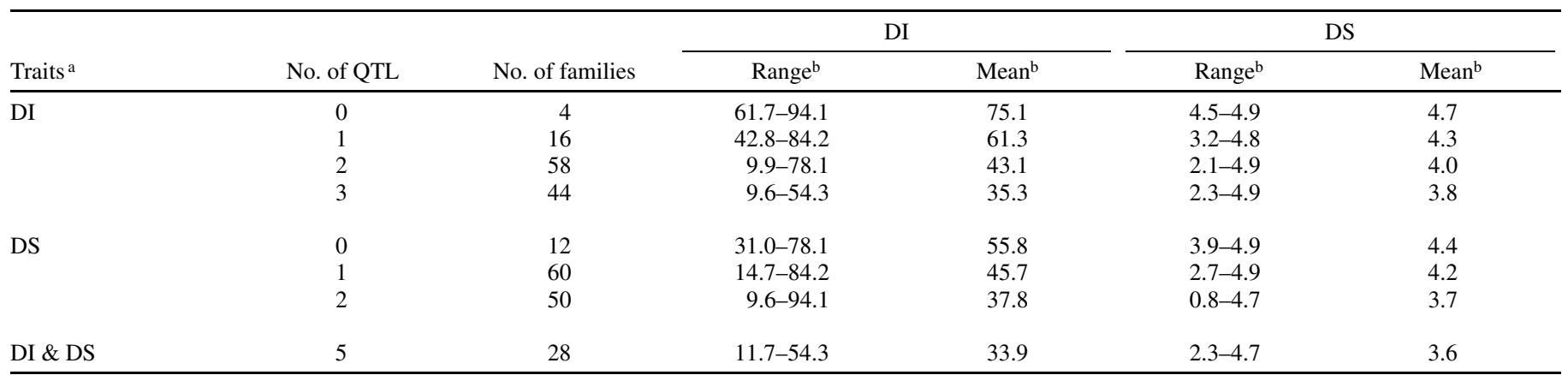

${ }^{a}$ DI and DS are disease incidence and disease severity, respectively.

${ }^{\mathrm{b}}$ Mean and range are the average of the three tests (Carrington, ND, in 2006; Carrington, ND, in 2007; Fargo, ND, in 2007).

vated sunflower. Each PCR reaction generated 9 polymorphic TRAP markers on average in the present mapping population, whereas only $20.5 \%$ of the polymorphisms were detected between the two parents with SSR markers. Therefore, TRAP is an efficient molecular marker technique for the construction of a linkage map, as suggested in previous studies $(6,7)$. TRAP markers have been used in linkage map construction in other plant species $(3,13,15)$. Moreover, TRAP takes the advantage of the annotated EST information to generate markers around the target sequence (6). The information of the EST flanking the QTL might provide useful information to facilitate gene cloning. For example, the TRAP markers flanking the two major QTL, QDi4 and QDs2, were designed against EST related to NBS (QHB18I19) and kinase (QHA11I24 and QHA10B18), respectively. These TRAP markers might be helpful for a better understanding and cloning of the resistance QTL in future studies.

\section{ACKNOWLEDGMENTS}

Mention of trade names or commercial products in this article is solely for the purpose of providing specific information and does not imply recommendation or endorsement by the US Department of Agriculture. We thank N. Balbyshev, A. Hogness, W. Ma, and P. Wang for technical assistance. This work was supported by a competitive grant from the National Sclerotinia Initiative and by USDA-ARS CRIS 5442-21000027-00D.

\section{LITERATURE CITED}

1. Bert, P. F., Dechamp-Guillaume, G., Serre, F., Jouan, I., Tourvieille de Labrouhe, D., Nicolas, P., and Vear, F. 2004. Comparative genetic analysis of quantitative traits in sunflower (Helianthus annuus L.) 3. Characterisation of QTL involved in resistance to Sclerotinia sclerotiorum and Phoma macdonaldi. Theor. Appl. Genet. 109:856-874.

2. Bert, P. F., Jouan, I., Tourvieille de Labrouhe, D., Serre, F., Nicolas, P., and Vear, F. 2002. Comparative genetic analysis of quantitative traits in sunflower (Helianthus annuus L.) 1. QTL involved in resistance to Sclerotinia sclerotinrum and Diaporthe helianthi. Theor. Appl. Genet. 105:985-993.

3. Chen, X., Faris, J. D., Hu, J., Stack, R. W., Elias, T. A., Elias, M., Kianian, S. F., and Cai, X. 2007. Saturation and comparative mapping of a major Fusarium head blight resistance QTL in tetraploid wheat. Mol. Breed. 19:113-124.

4. Gedil, M. A., Wye, C., Berry, S. T., Seger, B., Peleman, J., Jones, R., Leon, A., Slabaugh, M. B., and Knapp, S. J. 2001. An integrated RFLPAFLP linkage map for cultivated sunflower. Genome 44:213-221. 
5. Gianessi, L. P., Silvers, C., Sankula, S., and Carpenter, J. E. 2002. Plant biotechnology: Current and potential impact for improving pest management in U.S. agriculture. An analysis of 40 case studies. Fungal resistant sunflower. National Center for Food and Agricultural Policy. http://croplife.intraspin.co/BioTech/pdf.usp?id=76\&filename=108MainReport.pdf.

6. Hu, J., and Vick, B. A. 2003. Target region amplification polymorphism, a novel marker technique for plant genotyping. Plant Mol. Biol. Rep. 21:289-294.

7. Hu, J., Chen, J., Bervillé, A., and Vick, B. A. 2004. High potential of TRAP markers in sunflower genome mapping. Page 665-671 in: Proc. 16th International Sunflower Conference. International Sunflower Association, Paris.

8. Hu, J., Yue, B., and Vick, B. A. 2007. Integration of TRAP markers onto a sunflower SSR marker linkage map constructed from 92 recombinant inbred lines. Helia 30:25-36.

9. Lai, Z., Livingstone, K., Zou, Y., Church, S. A., Knapp, S. J., Andrews, J., and Rieseberg, L. H. 2005. Identification and mapping of SNPs from ESTs in sunflower. Theor. Appl. Genet. 111:1532-1544.

10. Lander, E. S., and Botstein, D. 1989. Mapping Mendelian factors underlying quantitative traits using RFLP linkage maps. Genetics 121:185-199.

11. Lander, E. S., Green, P., Abrahamson, J., Barlow, H., Daly, M., Lincoln, S., and Newbury, L. 1987. MAPMAKER: An interactive computer program for constructing genetic maps of experimental and natural populations. Genomics 1:174-175.

12. Lincoln, S. E., Daley, M. J., and Lander, E. S. 1993. Mapping genes controlling quantitative traits with MAPMAKER/QTL1.1: A tutorial and reference manual, 2nd ed. Whitehead Institute Technical Report, Cambridge, MA.

13. Liu, Z. H., Anderson, J. A., Hu, J., Friesen, T. L., Rasmussen, J. B., and Faris, J. D. 2005. A wheat intervarietal genetic linkage map based on microsatellite and target region amplified polymorphism markers and its utility for detecting quantitative trait loci. Theor. Appl. Genet. 111: 782-794.
14. Mestries, E., Gentzbittel, L., Tourvieille de Labrouhe, D., Nicolas, P., and Vear, F. 1998. Analyses of quantitative trait loci associated with resistance to Sclerotinia sclerotiorum in sunflowers (Helianthus annuus L.) using molecular markers. Mol. Breed. 4:215-226.

15. Miklas, P. N., Hu, J., Grünwald, N. J., and Larsen, K. M. 2006. Potential application of TRAP (targeted region amplified polymorphism) markers for mapping and tagging disease resistance traits in common bean. Crop Sci. 46:910-916

16. Miller, J. F, and Gulya, T. J. 2006. Registration of two restorer (RHA 439 and RHA 440) and one maintainer (HA 441) Sclerotinia tolerant oilseed sunflower germplasms. Crop Sci. 46:482-483.

17. Pérez-Vich, B., Berry, S. T., Velasco, L., Fernández-Martínez, J. M., Gandhi, S., Freeman, C., Heesacker, A., Knapp, S. J., and Leon, A. J. 2005. Molecular mapping of nuclear male sterility genes in sunflower. Crop Sci. 45:1851-1857.

18. Rönicke, S., Hahn, V., Vogler, A., and Friedt, W. 2005. Quantitative trait loci analysis of resistance to Sclerotinia sclerotiorum in sunflower. Phytopathology 95:834-839.

19. SAS Institute. 1999. SAS/STAT User's Guide, Releases: 8.2, 8.1, 8.0. SAS Institute, Inc., Cary, NC.

20. Tang, S., Yu, J. K., Slabaugh, M. B., Shintani, D. K., and Knapp, S. J. 2002. Simple sequence repeat map of the sunflower genome. Theor. Appl. Genet. 105:1124-1136.

21. Tang, S. X., Leon, A., Bridges, W. C., and Knapp, S. J. 2006. Quantitative trait loci for genetically correlated seed traits are tightly linked to branching and pericarp pigment loci in sunflower. Crop Sci. 46:721-734.

22. Wang, T., Xu, S. S., Harris, M. O., Hu, J., Liu, L., and Cai, X. 2006. Genetic characterization and molecular mapping of Hessian fly resistance genes derived from Aegilops tauschii in synthetic wheat. Theor. Appl. Genet. 113:611-618.

23. Yu, J. K., Tang, S., Slabaugh, M. B., Heesacker, A., Cole, G., Herring, M., Soper, J., Han, F., Chu, W. C., Webb, D. M., Thompson, L., Edwards, K. J., Berry, S., Leon, A. J., Olungu, C., Maes, N., and Knapp, S. J. 2003. Towards a saturated molecular genetic linkage map for cultivated sunflower. Crop Sci. 43:367-387. 